\title{
ON THE CONVERGENCE OF BERNSTEIN POLYNOMIALS FOR SOME UNBOUNDED ANALYTIC FUNCTIONS
}

\author{
P. C. TONNE
}

If $f$ is a function from $[0,1]$ to the complex numbers, $n$ is a positive integer, and $z$ is a complex number, then

$$
B_{n}^{f}(z)=\sum_{p=0}^{n} f(p / n) C_{n, p}(1-z)^{n-p} z^{p}
$$

and $B_{n}^{f}$ is the Bernstein polynomial for $f$ of order $n$. We prove the following:

Theorem. Suppose that $A$ is a complex sequence, $t>0, m$ is a nonnegative integer, $\left|A_{p}\right| \leqq t(p+1)^{m}$ for each nonnegative integer $p$, and $f$ is a function such that, for each complex number $z$ with $|z|<1, f(z)$ $=\sum_{p=0}^{\infty} A_{p} z^{p}$, and $f(1)$ is a complex number. Then the Bernstein polynomial sequence $B^{f}$ has limit $f$ on the unit disc and converges uniformly on each closed subset of the unit disc (the set of all complex numbers with modulus less than 1).

Bernstein polynomials were introduced by S. N. Bernstein [1] as explicit uniform polynomial approximations for functions continuous on an interval. Bernstein (see [2, p. 88]) demonstrated the convergence of Bernstein polynomials for functions analytic on some open sets containing $[0,1]$, and the author has a result [3, Theorem 1] from which it follows that if a function is the limit of a power-series about 0 which is absolutely convergent at 1 , then the Bernstein polynomials for the function converge uniformly to the function on the closure of the unit disc.

G. G. Lorentz [2, p. 28] considers the Bernstein polynomials for unbounded functions $g$ where there is a positive number $\alpha$ and an irrational number $c$ in $[0,1]$ such that, for each rational number $x$ in $[0,1], g(x)=|x-c|^{-\alpha}$ and shows that, for almost all irrational numbers $c$ in $[0,1]$, the corresponding Bernstein polynomials converge, and that for uncountably many irrational numbers $c$, the corresponding Bernstein polynomials are unbounded at each number in $[0,1]$ except 0,1 , and $c$. It seems that little else is known about Bernstein polynomials for unbounded functions.

Proof of Theorem. If $F$ is a function from $[0,1]$ to the complex plane, $F(1)=0, n$ is a positive integer, and $z$ is a complex number,

Presented to the Society, September 1, 1967; received by the editors July 20, 1968. 
then (using the binomial theorem and interchanging order of summation)

$$
\begin{aligned}
B_{n}^{F}(z)= & \sum_{p=0}^{n} z^{p} C_{n, p} \sum_{q=0}^{p}(-1)^{p+q} C_{p, q} F(q / n) \\
= & \sum_{p=0}^{n-1} z^{p} C_{n, p} \sum_{q=0}^{p}(-1)^{p+q} C_{p, q} F(q / n) \\
& +z^{n} \sum_{q=0}^{n-1}(-1)^{n+q} C_{n, q} F(q / n) .
\end{aligned}
$$

Using (1) and four lemmas, we demonstrate the convergence of the Bernstein polynomials for the special functions defined in the next paragraph. With this convergence we prove our theorem.

For each positive integer $m$ let $g_{m}$ be a function such that for each point $z$ in the unit disc $g_{m}(z)=1 /(1-z)^{m}$ and $g_{m}(1)=0$. For each positive integer $m$ and each nonnegative integer $p$, the $p$ th derivative of $g_{m}$ is $g_{m}^{(p)}$ and $g_{m}^{(p)}(0) / p !=C_{p+m-1, m-1}$.

By the mean value formula for divided differences or by Theorems $A$ and 0 of [3], we have

LEMMA 1. If $p$ is a nonnegative integer and $m$ is a positive integer, then

$$
\lim _{n \rightarrow \infty} C_{n, p} \sum_{q=0}^{p}(-1)^{p+q} C_{p, q} g_{m}(q / n)=C_{p+m-1, m-1} .
$$

Lемма 2. If $m$ is a positive integer and $n$ is an integer greater than the nonnegative integer $p$, then

$$
0<C_{n, p} \sum_{q=0}^{p}(-1)^{p+q} C_{p, q} g_{m}(q / n) \leqq(p+1)^{2 m-1} .
$$

Proof. Let $p$ be a nonnegative integer. For each nonnegative integer $m$ and each number $z$ greater than $p$, let $G(z)$ be $\prod_{j=0}^{p} 1 /(z-j)$ and $H_{m}(z)$ be $\sum_{j=0}^{p} 1 /(z-j)^{m}$. If $z>p$, then $-G^{\prime}(z)=G(z) H_{1}(z)$ and, for each nonnegative integer $m, H_{1}^{(m)}(z)=(-1)^{m} m ! H_{m+1}(z)$ and

$$
\begin{aligned}
(-1)^{m+1} G^{(m+1)}(z) & =(-1)^{m}\left(-G^{\prime}\right)^{(m)}(z)=(-1)^{m}\left(G \cdot H_{1}\right)^{(m)}(z) \\
& =(-1)^{m} \sum_{k=0}^{m} C_{m, k} G^{(k)}(z) H_{1}^{(m-k)}(z) \\
& =\sum_{k=0}^{m} C_{m, k}(-1)^{k} G^{(k)}(z)(m-k) ! H_{m-k+1}(z) .
\end{aligned}
$$

By partial-fractions methods or by induction, it is shown that if $z>p$ then 


$$
p ! G(z)=\sum_{q=0}^{p}(-1)^{p+q} C_{p, q} \frac{1}{z-q} .
$$

Then, by induction (differentiation), we see that, for each nonnegative integer $m$ and each number $z$ greater than $p$,

$$
(-1)^{m} \frac{p !}{m !} G^{(m)}(z)=\sum_{q=0}^{p}(-1)^{p+q} C_{p, q} \frac{1}{(z-q)^{m+1}} .
$$

Let $n$ be an integer greater than $p$.

$$
p ! C_{n+1, p}(n+1) G(n+1)=\frac{n+1}{n+1-p}<\frac{n}{n-p}=p ! C_{n, p} n G(n),
$$

and, therefore, $0<p ! C_{n, p} n G(n) \leqq p ! C_{p+1, p}(p+1) G(p+1)=p+1$.

Suppose that $m$ is a nonnegative integer such that if $k$ is a nonnegative integer not exceeding $m$ then

$$
0<(-1)^{k} \frac{p !}{k !} C_{n, p} n^{k+1} G^{(k)}(n) \leqq(p+1)^{2 k+1} .
$$

Then,

$$
\begin{aligned}
(-1)^{m+1} \frac{p !}{(m+1) !} C_{n, p} n^{m+2} G^{(m+1)}(n) & \\
= & \frac{1}{(m+1) !} \sum_{k=0}^{m} C_{m, k} k !\left\{(-1)^{k} \frac{p !}{k !} C_{n, p} n^{k+1} G^{(k)}(n)\right\} \\
& \cdot(m-k) ! n^{m-k+1} H_{m-k+1}(n) \\
\leqq & \frac{1}{(m+1) !} \sum_{k=0}^{m} m !(p+1)^{2 k+1} \sum_{j=0}^{p} \frac{n^{m-k+1}}{(n-j)^{m-k+1}} \\
\leqq & \frac{1}{m+1} \sum_{k=0}^{m}(p+1)^{m+k+1} \sum_{j=0}^{p}(p+1)^{m-k+1}=(p+1)^{2 m+3}
\end{aligned}
$$

From the first step above we see that

$$
0<(-1)^{m+1} \frac{p !}{(m+1) !} C_{n, p} n^{m+2} G^{(m+1)}(n) .
$$

So, if $m$ is a positive integer,

$$
\begin{aligned}
0<C_{n, p} \sum_{q=0}^{p}(-1)^{p+q} C_{p, q} g_{m}(p / n) & \\
= & C_{n, p} n^{m} \frac{p !}{(m-1) !}(-1)^{m-1} G^{(m-1)}(n) \leqq(p+1)^{2 m-1}
\end{aligned}
$$


LEMMA 3. If each of $m$ and $n$ is a positive integer then

$$
\left|\sum_{q=0}^{n-1}(-1)^{n+q} C_{n, q} g_{m}(q / n)\right| \leqq n^{m+1} .
$$

Proof. Let $m$ be a positive integer. Let $\phi$ be a function from $[0,1]$ to the numbers such that $\phi(0)=0$ and if $x$ is a positive number in $[0,1]$ then

$$
\phi(x)=x\left\{1+\sum_{p=1}^{m-1} \frac{(-1)^{p}}{p !}(\ln x)^{p}\right\} .
$$

$\phi$ is increasing (and continuous). $\phi(1)=1$.

$$
\left|\sum_{q=0}^{0}(-1)^{1+q} C_{1, q} g_{m}(q / 1)\right|=1 \leqq 1^{m+1}
$$

Suppose that $n$ is a positive integer and

$$
\left|\sum_{q=0}^{n-1}(-1)^{n+q} C_{n, q} g_{m}(q / n)\right| \leqq n^{m+1} .
$$

Then

$$
\begin{aligned}
&\left|\sum_{q=0}^{n}(-1)^{n+1+q} C_{n+1, q} g_{m}\left(\frac{q}{n+1}\right)\right| \\
&=(n+1)^{m}\left|\sum_{q=0}^{n}(-1)^{q+1} C_{n+1, q+1} \frac{1}{(q+1)^{m}}\right| \\
& \leqq(n+1)^{m}\left|\sum_{q=0}^{n-1}(-1)^{q+1} C_{n, q+1} \frac{1}{(q+1)^{m}}\right| \\
&+(n+1)^{m}\left|\sum_{q=0}^{n}(-1)^{q} C_{n, q} \frac{1}{(q+1)^{m}}\right| \\
&=(n+1)^{m}\left|\sum_{q=1}^{n}(-1)^{q} C_{n, q} \frac{1}{q^{m}}\right| \\
&+(n+1)^{m}\left|\sum_{q=0}^{n}(-1)^{q} C_{n, q} \int_{0}^{1} x^{q} d \phi(x)\right| \\
&= \frac{(n+1)^{m}}{n^{m}}\left|\sum_{q=0}^{n-1}(-1)^{n+q} C_{n, q} g_{m}(q / n)\right| \\
&+(n+1)^{m} \int_{0}^{1}(1-x)^{n} d \phi(x) \\
& \leqq(n+1)^{m} \cdot n+(n+1)^{m}=(n+1)^{m+1} .
\end{aligned}
$$


Without proof we state

Lemma 4. If each of $b, c$, and $d$ is a complex sequence, $\sum_{p=0}^{\infty}\left|c_{p}\right|$ converges, $\sum_{p=0}^{\infty}\left|c_{p} b_{p}\right|$ converges, and $M$ is a complex matrix such that, for each nonnegative integer $p, \lim _{n \rightarrow \infty} M_{n p}=d_{p}$, and $\left|M_{n p}\right| \leqq b_{p}$ for each positive integer $n$, then $\sum_{p=0}^{\infty}\left|c_{p} d_{p}\right|$ converges and

$$
\lim _{n \rightarrow \infty} \sum_{p=0}^{n-1} c_{p} M_{n p}=\sum_{p=0}^{\infty} c_{p} d_{p}
$$

From Lemma 4, Lemma 1, and Lemma 2 it follows that if $0<r<1$ and $m$ is a positive integer and $|z| \leqq r$ then

$$
\lim _{n \rightarrow \infty} \sum_{p=0}^{n-1} z^{p} C_{n, p} \sum_{q=0}^{p}(-1)^{p+q} C_{p, q} g_{m}(q / n)=\sum_{p=0}^{\infty} z^{p} C_{p+m-1, m-1}=g_{m}(z),
$$

and the convergence is uniform for $|z| \leqq r$, so that by (1) and Lemma $3 \lim _{n \rightarrow \infty} B_{n}^{g_{m}}(z)=g_{m}(z)$, the convergence being uniform for $|z| \leqq r$.

For our last digression, we define a matrix $Y$, very closely related to Sterling numbers of the first kind, such that if each of $p$ and $k$ is a nonnegative integer, then

$$
Y_{n k}=\sum_{q=0}^{p}(-1)^{p+q} C_{p, q} q^{k}
$$

(we interpret $0^{0}$ as 1 ) and note that $Y_{p+1, k+1}=(p+1)\left(Y_{p k}+Y_{p+1, k}\right)$; $Y_{p p}=p ! ; Y_{p k} \geqq 0 ; Y_{p k}=0$ for $p>k$; and, if $n$ is a positive integer $Y_{p, k+1} n^{-k-1} \geqq Y_{n k} n^{-k} ; \lim _{k \rightarrow \infty} Y_{n k} n^{-k}=1$; and, therefore, $Y_{n k} n^{-k} \leqq 1$.

Now, let $A, t, m$, and $f$ be as in the theorem.

Let $C$ be a closed subset of the unit disc. Let $r$ be a number less than 1 such that if $z$ is in $C$ then $|z| \leqq r$.

By (1), the power-series expansion for $g_{m+1}$ about 0 , and our definition for $Y$, we see that if $n$ is a positive integer, then

$$
B_{n}^{y_{m+1}}(r)=\sum_{p=0}^{n-1} r^{p} C_{n, p} \sum_{k=p}^{\infty} C_{k+m, m} n^{-k} Y_{p k}+r^{n} \sum_{q=0}^{n-1}(-1)^{n+q} C_{n, q} g_{m+1}(q / n),
$$

and

$$
\begin{aligned}
B_{n}^{g_{m+1}}(r)= & \sum_{p=0}^{n-1} r^{p} C_{n, p} C_{p+m, m} n^{-p} p !+\sum_{p=0}^{n-1} r^{p} C_{n, p} \sum_{k=p+1}^{\infty} C_{k+m, m} n^{-k} Y_{p k} \\
& +r^{n} \sum_{q=0}^{n-1}(-1)^{n+q} C_{n, q} g_{m+1}(q / n) .
\end{aligned}
$$


Now,

$$
\lim _{n \rightarrow \infty} B_{n}^{g_{m+1}}(r)=g_{m+1}(r)=\sum_{p=0}^{\infty} C_{p+m, m} r^{p},
$$

and, by Lemma 4 , as $n \rightarrow \infty$, the first term on the right side of (2) has the same limit, namely $\sum_{p=0}^{\infty} C_{p+m, m} r^{p}$, and the last term has limit 0 by Lemma 3 . Hence the limit as $n \rightarrow \infty$ of the second term is 0 , from which it follows that the second term on the right of (3), below, has limit 0 , since, for each nonnegative integer $k,\left|A_{k}\right| \leqq t(k+1)^{m}$ $\leqq t \cdot m ! \cdot C_{k+m, m}$.

If $n$ is a positive integer and $z$ is in $C$, then, by (1),

$$
B_{n}^{f}(z)=\sum_{p=0}^{n-1} z^{p} C_{n, p} \sum_{k=p}^{\infty} A_{k} n^{-k} Y_{p k}+z^{n} \sum_{q=0}^{n}(-1)^{n+q} C_{n, q} f(q / n),
$$

and

$$
\begin{aligned}
B_{n}^{f}(z)= & \sum_{p=0}^{n-1} z^{p} C_{n, p} A_{p} n^{-p} p !+\sum_{p=0}^{n-1} z^{p} C_{n, p} \sum_{k=p+1}^{\infty} A_{k} n^{-k} Y_{p k} \\
& +z^{n} \sum_{k=0}^{\infty} A_{k} n^{-k}\left\{Y_{n k}-n^{k}\right\}+z^{n} f(1) .
\end{aligned}
$$

In (3), as $n \rightarrow \infty$, the first term on the right, by Lemma 4 , has limit $\sum_{p=0}^{\infty} A_{p} z^{p}=f(z)$, and the second has limit 0 . Also, if $k$ is a nonnegative integer, $0 \geqq Y_{n k}-n^{k}$ and $\left|A_{k}\right| \leqq t \cdot m ! \cdot C_{k+m, m}$, and

$$
r^{n} \sum_{k=0}^{\infty} C_{k+m, m} n^{-k}\left\{Y_{n k}-n^{k}\right\}=r \sum_{q=0}^{n-1}(-1)^{n+q} C_{n, q} g_{m+1}(q / n),
$$

which by Lemma 3 has limit 0 as $n \rightarrow \infty$ so that the third term in (3) has limit 0 as $n \rightarrow \infty$. The fourth term in (3) has limit 0 as $n \rightarrow \infty$. The convergence is uniform for $z$ in $C$, and the theorem is proved.

\section{BIBLIOGRAPHY}

1. S. N. Bernstein, Démonstration du théorème de Weierstrass, fondée sur le calcul des probabilites, Commun. Soc. Math Kharkow (2), 13 (1912, 13), 1-2.

2. G. G. Lorentz, Bernstein polynomials, Univ. of Toronto Press, Ontario, 1953.

3. P. C. Tonne, Power-series and Hausdorff matrices, Pacific J. Math. 21 (1967), 189-198.

EMORY UNIVERSITY 